\title{
Effect of noise and detector sensitivity on a dynamical process: Inverse power law and Mittag-Leffler interevent time survival probabilities
}

\author{
Pensri Pramukkul, ${ }^{1,2}$ Adam Svenkeson, ${ }^{1}$ and Paolo Grigolini ${ }^{1}$ \\ ${ }^{1}$ Center for Nonlinear Science, University of North Texas, P.O. Box 311427, Denton, Texas 76203-1427, USA \\ ${ }^{2}$ Faculty of Science and Technology, Chiang Mai Rajabhat University, 202 Chang Phuak Road, Muang, Chiang Mai 50300, Thailand
}

(Received 16 October 2013; published 10 February 2014)

\begin{abstract}
We study the combined effects of noise and detector sensitivity on a dynamical process that generates intermittent events mimicking the behavior of complex systems. By varying the sensitivity level of the detector we move between two forms of complexity, from inverse power law to Mittag-Leffler interevent time survival probabilities. Here fluctuations fight against complexity, causing an exponential truncation to the survival probability. We show that fluctuations of relatively weak intensity have a strong effect on the generation of Mittag-Leffler complexity, providing a reason why stretched exponentials are frequently found in nature. Our results afford a more unified picture of complexity resting on the Mittag-Leffler function and encompassing the standard inverse power law definition.
\end{abstract}

DOI: 10.1103/PhysRevE.89.022107

PACS number(s): 02.50.-r, 89.75.-k, 05.40.-a

\section{INTRODUCTION}

Inverse power laws are generally interpreted as a signature of complexity, this being a so widely shared conviction as to make it difficult to afford a fair list of key references in a regular research paper. In this article we focus on power laws connected to temporal complexity and intermittent dynamics of critical fluctuations. As shown by Contoyiannis et al. [1], these fluctuations have a close connection with the intermittency generating maps [2-6]. The Pomeau-Manneville (PM) map generates sporadic randomness and the laminar regions between two consecutive randomness bursts have a distribution density that is an inverse power law. This is what we mean by temporal complexity, the theoretical perspective adopted in this article.

In real physical systems the inverse power law distribution densities are truncated in the long-time regime [7]. If fluctuations characterized by a truncated inverse power law distribution are used to generate diffusive motion, according to the central limit theorem the resulting diffusion process is ordinary. However, the time necessary to produce ordinary diffusion is much more extended compared to when the fluctuations are taken from a distribution without the scale-free property [7]. This important result can be interpreted as an explanation of why Lévy processes are frequently observed in nature. A weak noise truncating complex fluctuations is responsible for the emergence of normal diffusion in the longtime limit $[8,9]$. Although, moving from the one-dimensional to high-dimensional cases may generate much more complex effects [10,11], we refer to the one-dimensional case, where the effect of fluctuations perturbing the generators of anomalous diffusion is a transition in the long-time limit from anomalous to ordinary diffusion $[12,13]$.

The Mittag-Leffler (ML) function, a generalization of the exponential function, can display inverse power law behavior in the asymptotic limit. As a consequence, many researchers have focused their attention on the ML function as a manifestation of complexity, for example, in complex materials $[14,15]$. It must be pointed out that the ML function is becoming important also for its deep connections with the subject of fractional calculus [16]. There are indications that the ML function may emerge at criticality [17]. In spite of the increasing interest for this form of temporal complexity, there is no understanding about what the role of noise may be in this case.

It is important to notice that the cooperation-induced emergence of ML complexity [18,19] is suggesting a quite different connection between the complexity generating maps and noise. In fact, in both the case of random growth of surfaces and neural dynamics the single units in the absence of cooperation are random and as an effect of cooperation are forced to generate global properties where the influence of randomness is quenched. As a consequence, the stochastic force that the authors of Refs. [12,13] assumed to be of environmental origin, in the case of cooperation-induced complexity is the residual of the randomness of the single individuals. We make the reasonable conjecture that the truncation of the inverse power law in the case of the PM map is correctly predicted using the methods of Refs. [12,13], the internal rather than the environmental origin of fluctuations being irrelevant. However, this assumption does not lead us to the solution of the problem, because, to the best of our knowledge, there are no maps generating ML complexity in the same way as the PM map generates the inverse power law distributions.

To settle this problem we rest on the theoretical result of Ref. [20]. The authors of this article showed that ML complexity can be derived from the PM map assuming that the events generated by the PM map have a very small probability $P_{S}$ of being detected. When the probability of perceiving another PM map event after the occurrence of a visible event is very small, the decay of the corresponding survival probability becomes extremely slow. A rescaling procedure is applied to go back to an appropriate time scale. After rescaling, the resulting survival probability is a ML function. This entire process was named the "stochastic central limit theorem" (SCLT) by the authors in Ref. [20].

We make the assumption that the effect of noise on ML complexity can be determined by applying the SCLT to the PM map perturbed by noise according to the prescriptions of Refs. $[12,13]$. The main result of this method is that the 
ML function is much more sensitive to either internal or environmental noise than the PM map survival probability.

This paper is organized as follows. In Sec. II we show the emergence of the noise-induced truncation in the inverse power law, PM case. The effect of noise on the ML function is described via numerical calculations in Sec. III. We devote Sec. IV to explaining theoretically the results of the numerical calculations and Sec. V to concluding remarks.

\section{ORDINARY INVERSE POWER LAW AND NOISE}

We make the simplifying assumption that the cooperationinduced temporal complexity is satisfactorily described by the idealized PM map [21,22]. It is important to notice, however, that the idealized PM map is a generator of complex events, namely of an inverse power law only in the long time limit. The PM map generates a survival probability with a transition from the microscopic to the macroscopic time that is absent in the ML complexity. To derive the ML process from the PM dynamics we have to find a way to annihilate the events carrying information on the microscopic PM dynamics, this being the central property of this article.

This section to a large extent is a review of the work done in Refs. [12,13] that we do to make the paper self-contained and to make it easier for the readers to understand the procedure that we adopt to solve the problem in the case of ML complexity.

The idealized PM map reads [21,22]

$$
\frac{d}{d t} y=a y^{z}
$$

with $z>1$. The variable $y$ is assigned the initial condition $y_{0}$ obeying the inequality $0<y_{0}<1$. When it reaches the value $y=1$ it is injected back with uniform probability, signifying the occurrence of an intermittent event in a complex system.

The time distance between the back injection to an initial value $y_{0}$, that for simplicity's sake we now call $y$, and the arrival at the border, through integration of Eq. (1), is

$$
\tau=\frac{\mu-1}{a}\left\{\frac{1}{y^{\frac{1}{\mu-1}}}-1\right\} .
$$

By making the choice of the initial value $y$ uniform, we find that the time distance between two consecutive events is given by the waiting time distribution density

$$
\psi(\tau)=(\mu-1) \frac{T^{\mu-1}}{(\tau+T)^{\mu}},
$$

where

$$
\mu \equiv \frac{z}{z-1}
$$

and

$$
T \equiv \frac{\mu-1}{a} .
$$

The mean waiting time $\langle\tau\rangle$ is given by

$$
\langle\tau\rangle=\frac{T}{(\mu-2)},
$$

for $\mu>2$. When $\mu<2$, the mean time diverges and the process generates aging and ergodicity breaking [23].
The survival probability $\Psi(t)$ corresponding to the waiting time distribution density $\psi(\tau)$ of Eq. (3) reads

$$
\Psi(t)=\left(\frac{T}{T+t}\right)^{\mu-1} .
$$

This analytical expression allows us to explain the main difference between the PM and the ML complexity. Using the widely accepted conviction that complexity implies an inverse power law, we can say that the PM dynamics are complex for times $t \gg T$, where the waiting time distribution density $\psi(\tau)$ of Eq. (3) becomes indistinguishable from an inverse power law, due to the property

$$
\lim _{\tau \rightarrow \infty} \psi(\tau) \propto \frac{1}{\tau^{\mu}} .
$$

On the contrary, for the ML survival probability,

$$
\Psi(t)=E_{\alpha}\left[-(\lambda t)^{\alpha}\right],
$$

complexity emerges at even extremely short times, because [14] in the short-time regime $(t \ll 1 / \lambda)$ the ML survival probability is the stretched exponential, $\exp \left[\frac{-(\lambda t)^{\alpha}}{\Gamma(\alpha+1)}\right]$, where $\Gamma$ denotes the gamma function. In the long-time limit $(t \rightarrow \infty)$, the ML survival probability becomes proportional to $1 / t^{\alpha}$. Thus, if we identify it with the asymptotic time of the PM map survival probability of Eq. (7) we must set the condition

$$
\alpha=\mu-1 \text {. }
$$

In conclusion, the short-time stretched exponential affords information on the complexity of the process.

Note that for the ML function to be compatible with the definition of survival probability the condition $\alpha<1$ is required, thereby implying $\mu<2$. According to the earlier argument, $\langle\tau\rangle$ diverges leading to aging and ergodicity breaking [23].

Following the earlier work of Refs. [12,13], adapted to the idealized PM map of Refs. [21,22], we now assume that the PM map is perturbed by noise according to the prescription

$$
\frac{d}{d t} y=a y^{z}+f(t)
$$

The fluctuation is a white noise given by $f(t)=\sqrt{\sigma} r(t)$, where $r(t)$ is either 1 or -1 , according to a coin-tossing prescription. Note that in this article we adopt arbitrary units resting on the assumption that the numerical integration time step is equal to the time unit, $\Delta t=1$. As a consequence of this assumption, for the integration of Eq. (11) we obtain the correlation function

$$
\langle f(n) f(m)\rangle=2 \sigma \delta_{n, m} .
$$

We refer to $\sigma$ as the noise intensity.

Figure 1 shows that the main effect of $f(t)$ is that of an exponential truncation to the survival probability $\Psi(t)$ of Eq. (7). We see that the larger the noise intensity $\sigma$, the shorter the truncation time $t_{T}$, the time at which the transition from inverse power law to exponential behavior occurs. In fact, we estimate that the noise intensities $1 \times 10^{-2}, 1 \times 10^{-6}$, and $1 \times 10^{-10}$ approximately make $t_{T}$ equal to 126,5372 , and 233258 , respectively.

These results qualitatively agree with those of Refs. [12,13]. Here we make the evaluation of the truncation time more accurate than in these earlier papers and at the same time 


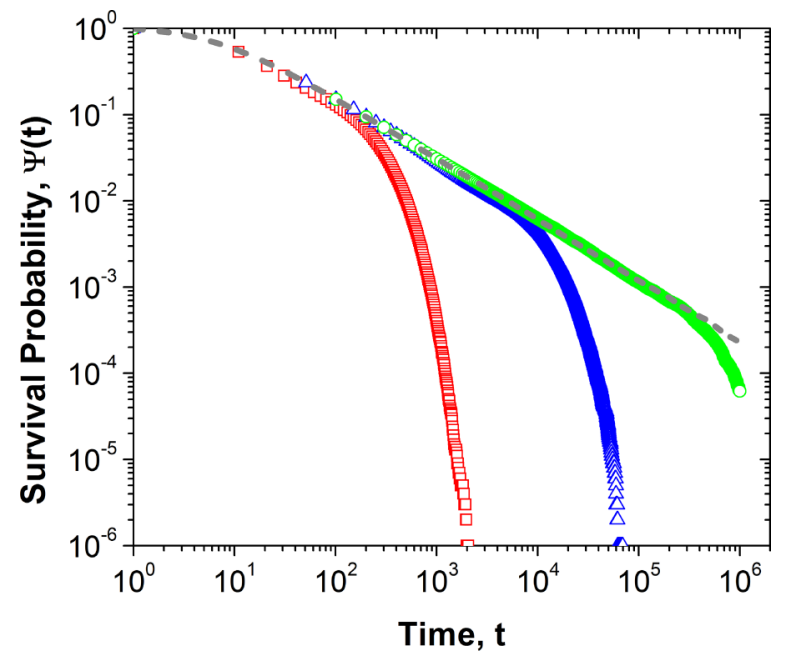

FIG. 1. (Color online) Survival probability of the PM map under the influence of fluctuations, Eq. (11), with $\mu=1.7$ and $T=7$ ( $a=$ 0.1 and $z=2.428$ ), and noise intensities $\sigma=1 \times 10^{-2}$ (red squares), $\sigma=1 \times 10^{-6}$ (blue triangles), $\sigma=1 \times 10^{-10}$ (green circles). The dashed gray line refers to the survival probability of Eq. (7) corresponding to the PM map without noise, Eq. (1).

we connect $t_{T}$ to the lifetime of the truncating exponential function, $1 / \epsilon$. We make the following assumption

$$
\Psi_{\epsilon}(t)= \begin{cases}\left(\frac{T}{T+t}\right)^{\mu-1}, & \text { if } t<t_{T} \\ \left(\frac{T}{T+t_{T}}\right)^{\mu-1} e^{-\epsilon\left(t-t_{T}\right)}, & \text { if } t \geqslant t_{T} .\end{cases}
$$

We adopt a log-linear representation to fit the exponential tail and $a \log -\log$ representation to fit the inverse power law region, thereby generating the dashed line and the dotted line of Fig. 2, respectively. Then, in the linear representation, for any time $t$ we evaluate the distance between the dotted and the dashed

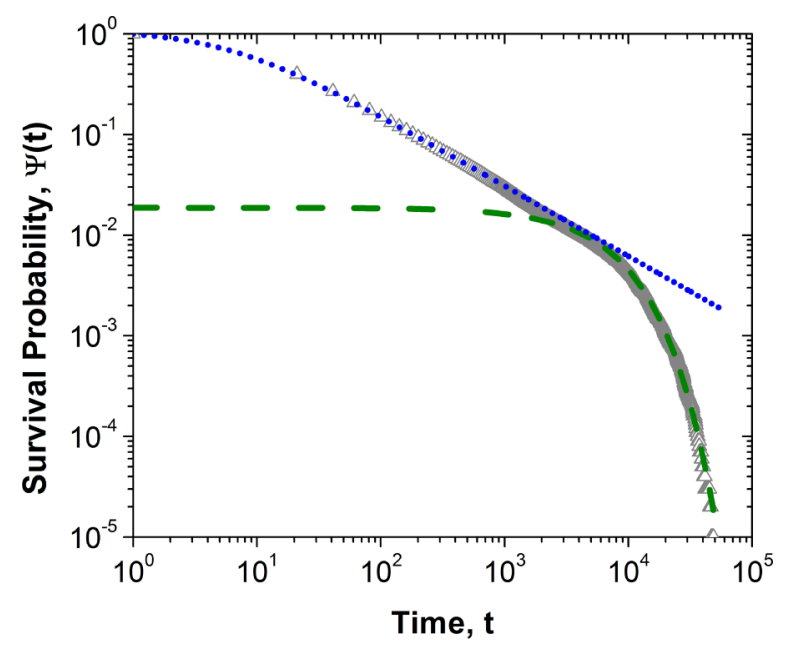

FIG. 2. (Color online) Fitting procedure for the perturbed PM map with $\sigma=1 \times 10^{-6}$ (gray triangles) with the inverse power law $\left(\frac{T}{T+t}\right)^{\mu-1}$ (blue dotted line) and exponential decay $e^{-\epsilon t}$ (green dashed line), yielding $T=7$ and $\epsilon=1.425 \times 10^{-4}$, respectively. The truncation time $t_{T}$ is the time where there is the smallest gap between the inverse power law and exponential decay. For this case $t_{T}=5372$.

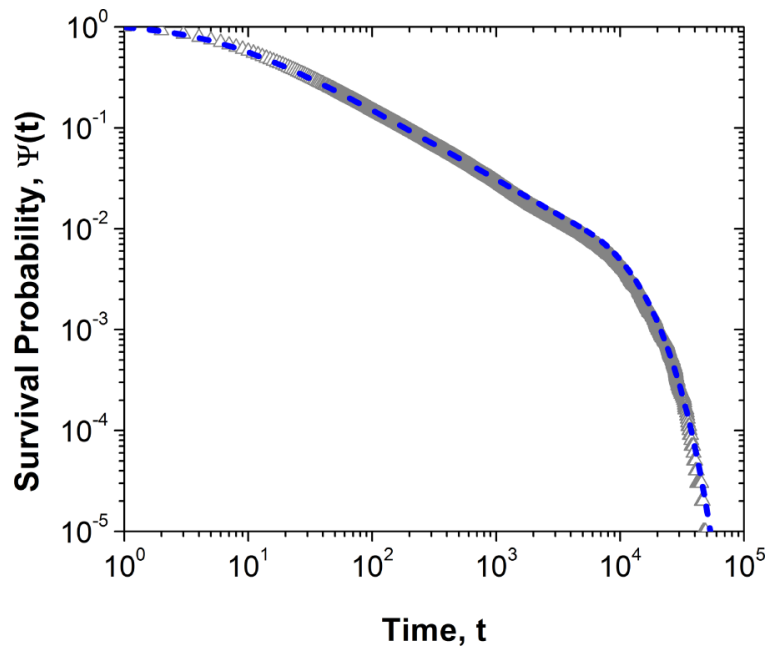

FIG. 3. (Color online) Comparison of the survival probability for the perturbed PM map with $\sigma=1 \times 10^{-6}$ (gray triangles) and the analytical expression of Eq. (13) (blue dashed line) using fitting parameters $t_{T}=5372$ and $\epsilon=1.425 \times 10^{-4}$ given by the fitting procedure described in Fig. 2.

line, and we define $t_{T}$ as the time generating the minimal distance. We record also the values of $\epsilon$. For instance, the fit of the exponential tail in the log-linear representation, in the correspondence of $\sigma=1 \times 10^{-2}, 1 \times 10^{-6}$, and $1 \times 10^{-10}$, which are the noise intensities used in Fig. 1, yields for $\epsilon$ the values $6.32 \times 10^{-3}, 1.43 \times 10^{-4}$, and $3.09 \times 10^{-6}$, respectively. The result illustrated in Fig. 3, which plots Eq. (13) for $\sigma=1 \times 10^{-6}$ using the values of $t_{T}$ and $\epsilon$ obtained using this fitting procedure, gives a good agreement with the numerical calculation.

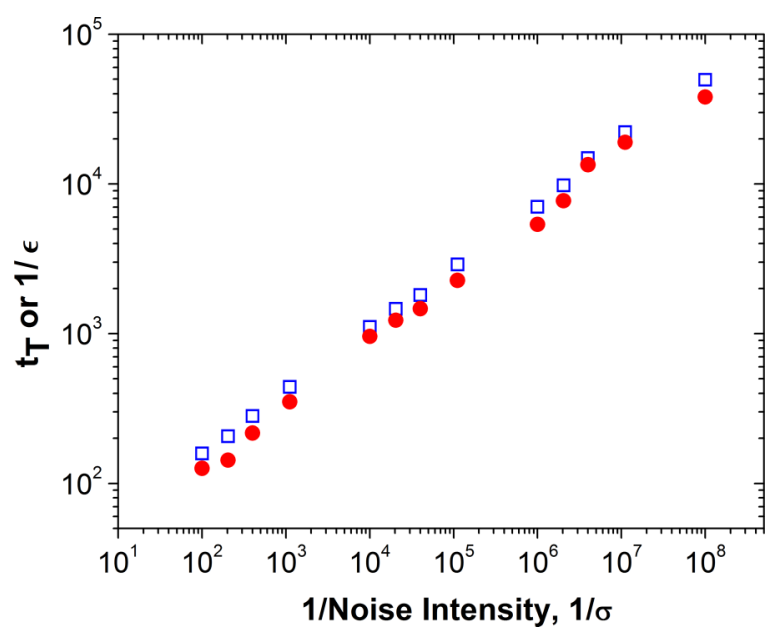

FIG. 4. (Color online) Comparing the truncation time, $t_{T}$ (red circles), and the lifetime of the truncating exponential function $1 / \epsilon$ (blue squares), at different values of noise intensity for the perturbed PM map with $\mu=1.7$. The slopes of $t_{T}$ and $1 / \epsilon$ are 0.4255 and 0.4204 , respectively. 
It is remarkable that $t_{T}$ is very close to $1 / \epsilon$, as shown in Fig. 4. As a consequence, the analytical expression

$$
\Psi_{\epsilon}(t)=\left(\frac{T}{T+t}\right)^{\mu-1} e^{-\epsilon t}
$$

is very close to the prescription of Eq. (13) for both $t<t_{T}$ and $t>t_{T}$. This simpler expression will be used for the theoretical arguments in Sec. IV.

Using Eq. (2), it is possible to predict the dependence of $t_{T}$ on the noise intensity $\sigma$. In fact, we see that the initial conditions very close to the origin generate extremely large waiting times. This is so because the dynamics of $y$ generated by Eq. (1) are extremely slow in that region. The noise $f(t)$ of Eq. (11) generates diffusion, and the diffusion process in the region very close to $y=0$ is much faster than the deterministic dynamics. As a consequence, there exists a region $0<y<\delta$, with $\delta$ very small, which does not contribute to Eq. (2). The trajectories with an initial condition in this region diffuse very quickly beyond the border. This effect generates the truncation time $t_{T}$ given by

$$
t_{T} \approx \frac{\mu-1}{a}\left\{\frac{1}{\delta^{\frac{1}{\mu-1}}}-1\right\} .
$$

With a rigorous theoretical treatment, the authors of Ref. [13] established the relation between $\delta$ and the noise intensity to be

$$
\delta \approx \sigma^{\frac{\mu-1}{2 \mu-1}} .
$$

This result, using Eq. (15), yields

$$
t_{T} \propto \sigma^{-\frac{1}{2 \mu-1}} .
$$

We check the accuracy of this prediction through numerical calculations yielding results that are illustrated in Fig. 5. It is important to notice that although Eq. (15) is valid for $\mu$ ranging from 1 to $\infty$, it has a special relevance in the region $1<\mu<2$, because in this specific region the mean time $\langle\tau\rangle$ would be divergent with $t_{T}=\infty$, but instead it is made finite by the exponential truncation due to the influence of noise.

\section{THE MITTAG-LEFFLER FUNCTION AFFECTED BY NOISE}

The complex system generating events via the PM map yields the survival probability of Eq. (7). Let us assume that only some of these events are detected, the probability of detecting one event being $P_{S}<1$. Then the resulting survival probability reads [20]

$$
\Psi_{V}(t)=\sum_{n=0}^{\infty}\left(1-P_{S}\right)^{n} \int_{0}^{t} d t^{\prime} \psi_{n}\left(t^{\prime}\right) \Psi\left(t-t^{\prime}\right),
$$

where $\psi_{n}(t) d t$ is the probability that the $n$th event occurs at time $t$, being the last of a sequence of $n-1$ events occurring earlier. Note $\psi_{1}(t)$ is $\psi(t)$, the waiting time distribution defined in Eq. (3), and $\Psi(t)$ is the corresponding survival probability in Eq. (7). The Laplace transform of Eq. (18) is

$$
\hat{\Psi}_{V}(u)=\frac{1}{u+\hat{\Phi}(u) P_{S}},
$$

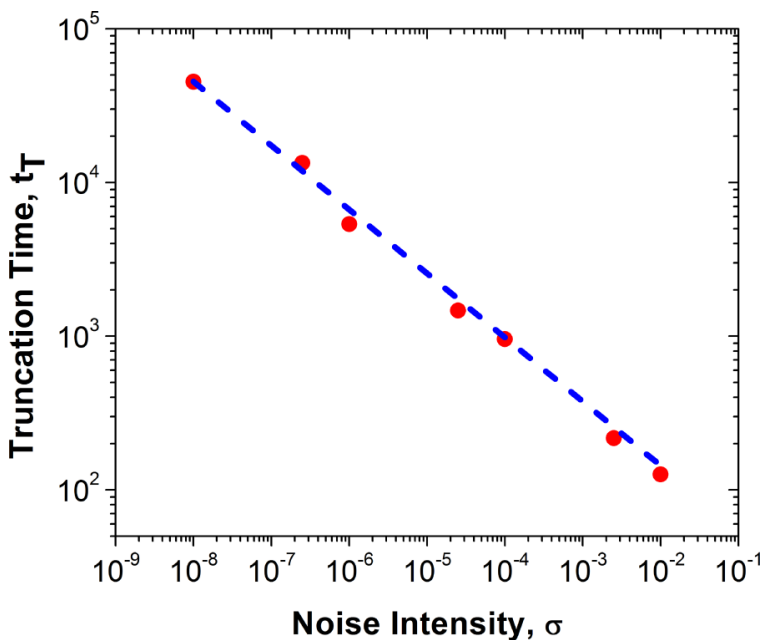

FIG. 5. (Color online) The numerical results for the dependence of the truncation time on the noise intensity for the perturbed PM map ( $\mu=1.7)$ (red circles) fit the theoretical prediction (blue dashed line) given by Eq. (17). The slope of the numerical data, -0.428 , corresponds to the exponent in Eq. (17), -0.416 .

where $\hat{\Phi}(u)$ is the Laplace transform of the Montroll-Weiss memory kernel [20],

$$
\hat{\Phi}(u)=\frac{u \hat{\psi}(u)}{1-\hat{\psi}(u)} .
$$

Equation (19) can be written in the form

$$
\hat{\Psi}_{V}(u)=\frac{1}{u+\lambda^{\alpha}\left(u^{1-\alpha}+\Sigma(u)\right) P_{S}} .
$$

We denote by $\Sigma(u)$ the corrections to the ML function Laplace transform,

$$
\hat{\Psi}(u)=\frac{1}{u+\lambda^{\alpha} u^{1-\alpha}},
$$

generated by the adoption of the PM waiting time distribution density and survival probability. The connection to the PM map requires that

$$
T=\frac{1}{\lambda[\Gamma(1-\alpha)]^{\frac{1}{\alpha}}} .
$$

Details on the higher-order corrections in Eq. (21) can be found in the Appendix.

When $P_{S}$ is small enough the resulting $\Psi_{V}(t)$ is indistinguishable from the ML function $E_{\alpha}\left[-(\Lambda t)^{\alpha}\right]$ with

$$
\Lambda=\lambda P_{S}^{1 / \alpha} \text {. }
$$

As shown in Ref. [20], the emergence of an exact ML function with $P_{S} \rightarrow 0$ is made compelling by compressing the time scale, namely, either by replacing $u$ with $u=u^{\prime} P_{S}^{1 / \alpha}$ (in the Laplace domain) or $t$ with $t^{\prime}=t P_{S}^{1 / \alpha}$ (in the time domain). This has the effect of making $\Sigma\left(u^{\prime}\right)$ vanish. In Fig. 6, in fact, after rescaling, the resulting survival probability with $P_{S}=$ 0.01 goes close to the ideal ML function generated using the algorithm in Ref. [24], while the rescaling of the resulting survival probability with $P_{S}=0.5$ goes in between the original PM map and the ideal ML function. In conclusion, the ML 


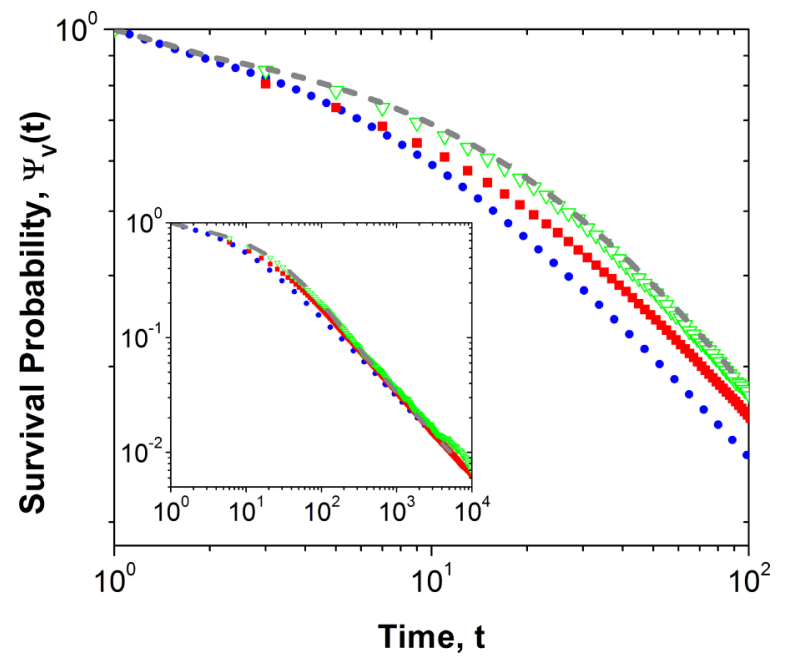

FIG. 6. (Color online) The survival probability after applying the SCLT to the PM map with $P_{S}=0.5$ (red squares) and $P_{S}=0.01$ (green triangles). Decreasing $P_{S}$ causes a shift in the short-time region of the survival probability from the original PM map $\left(P_{S}=1\right)$ (blue dotted line) toward the ideal ML function (gray dashed line). The numerical survival probability coincides with the ideal ML function when $P_{S}=0.01$ for $\alpha=0.7$. The inset shows that the asymptotic time behavior remains unchanged.

function can be generated numerically by the rescaling process of the PM map with a small probability of event detection, that is, by the SCLT procedure.

These results suggest the following solution to the problem of evaluating the effects of noise on the emergent ML function. We run Eq. (11), with $T$ related to $\lambda$ through Eq. (23) and $z=(1+\alpha) / \alpha$, and with the condition that not all the event times are recorded, but only a fraction of them with probability $P_{S}$. We adopt for $P_{S}$ the value that generates after rescaling a very good agreement with the exact ML prescription, $P_{S}=0.01$ in the case of Fig. 6 , and we scale back the result. Of course, using this procedure, we find that like in the PM map, the noise causes an exponential truncation of the inverse power law tail of the ML survival probability as shown in Fig. 7. However, the truncation occurs much earlier than in the perturbed PM map of Fig. 1. For example, with $P_{S}=0.01$ and the noise intensity $\sigma=1 \times 10^{-10}$, the truncation time of the perturbed ML function (in Fig. 7) corresponding to $\alpha=0.7$ is approximately 500 times smaller than the truncation time of the perturbed PM map. Due to the fact that the noise has a greater effect on the ML survival probability than the PM map, we reach the conclusion that the stretched exponentials frequently found in nature may be ML functions with their fat tails hidden by noise.

It is important to notice that assigning very small values to $P_{S}$ is equivalent to diminishing the dependence of the resulting dynamical process on the microscopic events generated by the PM map, and this is subtly related to the sensitivity of the ML process to noise. We shall devote Sec. IV to a discussion of this important issue. Here we limit ourselves to illustrate additional numerical results that in Sec. IV will help the realization of this rigorous criterion. We find $t_{T}$ and $\epsilon$ of the perturbed ML function numerically using the same procedure as in Sec. II,

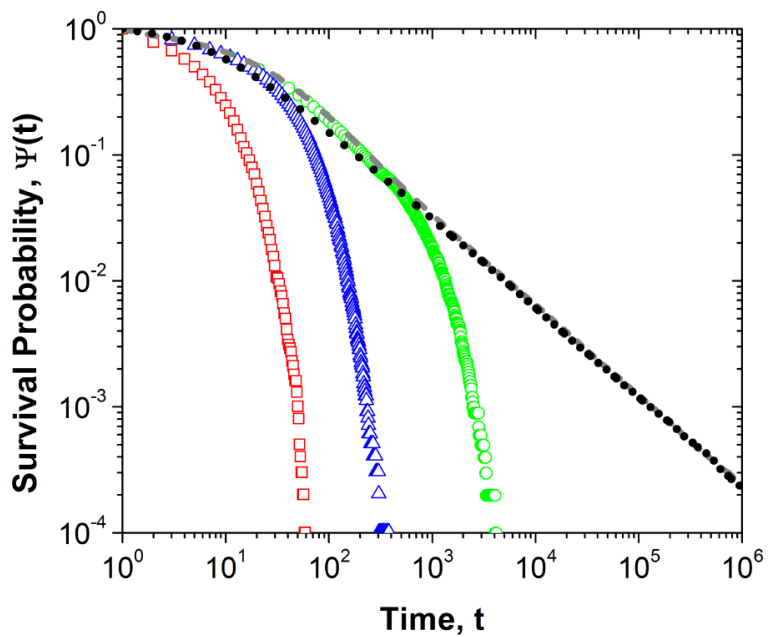

FIG. 7. (Color online) The perturbed ML function generated by applying the SCLT (with $P_{S}=0.01$ ) to the perturbed PM map with $\sigma=1 \times 10^{-2}$ (red squares), $\sigma=1 \times 10^{-6}$ (blue triangles), and $\sigma=1 \times 10^{-10}$ (green circles). This is compared to both the ideal ML function (gray dashed line) and the PM map without noise (black dotted line). The strong effect of noise on the generation of the perturbed ML function prevents the realization of the asymptotic inverse power law behavior.

based on the assumption

$$
\Psi_{\epsilon}(t)= \begin{cases}E_{\alpha}\left[-(\lambda t)^{\alpha}\right], & \text { if } t<t_{T} \\ E_{\alpha}\left[-\left(\lambda t_{T}\right)^{\alpha}\right] e^{-\epsilon\left(t-t_{T}\right)}, & \text { if } t \geqslant t_{T} .\end{cases}
$$

The result is shown in Fig. 8 for $\sigma=1 \times 10^{-10}$, where we can see the assumption in Eq. (25) again gives a very good agreement with the numerical calculation. On the basis of these numerical results we conclude that also in this case

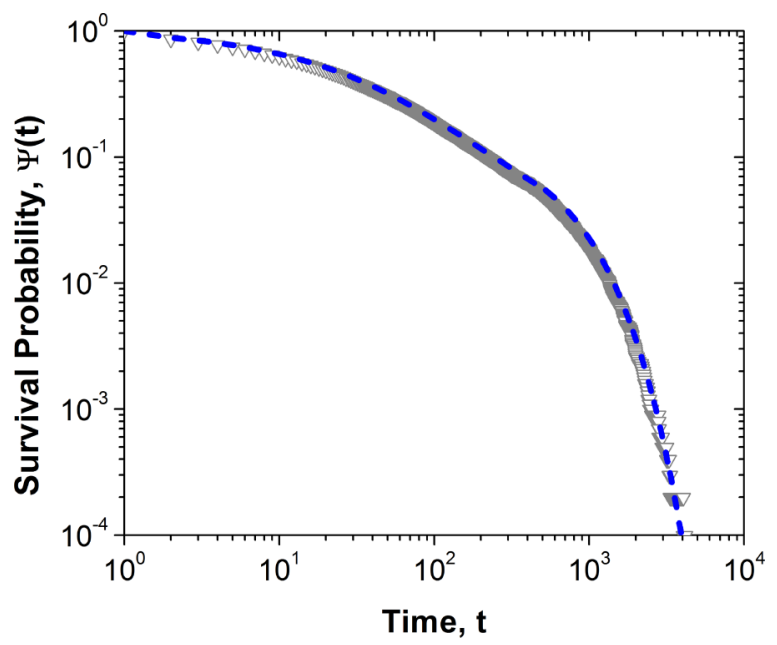

FIG. 8. (Color online) Comparison of the survival probability for the perturbed ML function with $\sigma=1 \times 10^{-10}$ (gray triangles) and the analytical expression of Eq. (25) (blue dashed line) using fitting parameters $t_{T}=524$ and $\epsilon=1.847 \times 10^{-3}$ given by the same fitting procedure described in the legend of Fig. 2. 
$\epsilon \approx \frac{1}{t_{T}}$, with the condition, however, that $t_{T}$ is much smaller than the PM truncation time.

\section{THEORETICAL ARGUMENTS}

The main purpose of this section is to explain the connection between a real ML function, namely, a ML dynamical process realized using a detector of events of limited sensitivity, and the accuracy of its representation through an ideal ML function, generated by the rigorous mathematical prescriptions of Refs. [14-16].

\section{A. Detection of events in the presence of noise}

As pointed out earlier, to study the influence of noise on a ML process defined by $\lambda$ and $\alpha$, we run the PM map prescription of Eq. (11) with $T$ given by Eq. (23) and $z=(1+\alpha) / \alpha$. We adopt the analytical expression of Eq. (14), and detecting the events with a given $P_{S}$ we obtain

$$
\hat{\Psi}_{\epsilon}(u)=\frac{1}{u+\epsilon+\lambda^{\alpha}\left[(u+\epsilon)^{1-\alpha}+\Sigma(u+\epsilon)\right] P_{S}} .
$$

The rescaling $u=u^{\prime} P_{S}^{1 / \alpha}$ turns Eq. (26) into

$$
\hat{\Psi}_{\epsilon}\left(u^{\prime}\right)=\frac{1}{u^{\prime} P_{S}^{1 / \alpha}+\epsilon+\lambda^{\alpha}\left[\left(u^{\prime} P_{S}^{1 / \alpha}+\epsilon\right)^{1-\alpha}+\Sigma\left(u^{\prime} P_{S}^{1 / \alpha}+\epsilon\right)\right] P_{S}} .
$$

It is convenient to write Eq. (27) as

$$
\hat{\Psi}_{\epsilon}\left(u^{\prime}\right)=\frac{1}{P_{S}^{1 / \alpha}\left\{u^{\prime}+\epsilon P_{S}^{-1 / \alpha}+P_{S}^{1-1 / \alpha} \lambda^{\alpha}\left[\left(u^{\prime} P_{S}^{1 / \alpha}+\epsilon\right)^{1-\alpha}+\Sigma\left(u^{\prime} P_{S}^{1 / \alpha}+\epsilon\right)\right]\right\}} .
$$

The overall factor $\frac{1}{P_{S}^{1 / \alpha}}$ can be canceled so as to fit the normalization condition $\Psi(0)=1[20]$, and $u^{\prime}$ replaced by $u$, thereby leading to

$$
\hat{\Psi}_{\epsilon}(u)=\frac{1}{u+\epsilon P_{S}^{-1 / \alpha}+P_{S}^{1-1 / \alpha} \lambda^{\alpha}\left[\left(u P_{S}^{1 / \alpha}+\epsilon\right)^{1-\alpha}+\Sigma\left(u P_{S}^{1 / \alpha}+\epsilon\right)\right]} .
$$

Notice that in the absence of noise $(\epsilon=0)$ all the contributions to this expression except $\Sigma$ become independent of $P_{S}$. The correction term vanishes for $P_{S} \rightarrow 0$. When noise is present, the adoption of this prescription would make Eq. (29) always generate a sharp exponential for $P_{S} \rightarrow 0$. As we shall see in Sec. IV B, we have to set a bottom limit to $P_{S}$.

Let us use Eq. (29) to evaluate the mean waiting time $\langle\tau\rangle$, which is obtained by setting $u=0$. By neglecting the contribution generated by $\Sigma$, we get

$$
\langle\tau\rangle=\frac{P_{S}^{1 / \alpha}}{\epsilon^{1-\alpha}} \frac{1}{\epsilon^{\alpha}+\lambda^{\alpha} P_{S}} .
$$

Comparing the ML function case with $P_{S}<1$ to the PM map, with $P_{S}=1$, we obtain

$$
\frac{\langle\tau\rangle_{\mathrm{ML}}}{\langle\tau\rangle_{\mathrm{PM}}}=P_{S}^{1 / \alpha} \frac{\epsilon^{\alpha}+\lambda^{\alpha}}{\epsilon^{\alpha}+\lambda^{\alpha} P_{S}}
$$

The adoption of this formula with the numerical values adopted in Sec. III leads to results in reasonable agreement with the numerical results of that section. In fact, the ratio of the ML to the PM mean waiting times for $\sigma=1 \times 10^{-10}$ evaluated from Eq. (31) is 0.120, which is in reasonable agreement with the ratio from the numerical calculation, 0.138. It is important to notice that $\langle\tau\rangle \approx t_{T}^{1-\alpha}$. Thus, the ratio of the ML function truncation time to the PM truncation time is of the order of $0.138^{3.333}=0.00135$, whose order of magnitude is the same as that observed in Sec. III for $P_{S}=0.01$.

\section{B. Accuracy of the procedure adopted to derive ML function from PM map}

To determine the proper value of $P_{S}$ in Eq. (31) necessary to achieve the desired level of accuracy in the ML process, we move from Eq. (21) to

$$
\hat{\Psi}(u)=\frac{1}{u+\Lambda^{\alpha} u^{1-\alpha}},
$$

where $\Lambda$ is defined in Eq. (24) and we proceed as follows. We define $P_{S}$ in such a way as to make the correction $\Sigma(u)$ of Eq. (21) as small as possible. From the expression of $\Sigma(u)$ in Eq. (A7), the lowest-order contributions to $\Sigma(u)$ are given by $\Sigma(u) \propto u^{\beta}$ with $\beta=1$ for $0<\alpha<0.5$, and $\beta=2-2 \alpha$ for $0.5 \leqslant \alpha<1$. To be more precise, we must compare the correction $\Sigma(u)$ to the leading contribution $u^{1-\alpha}$ after applying the rescaling $u=u^{\prime} P_{S}^{1 / \alpha}$. We assume $u^{\prime}$ to be of the order of unity and the accuracy of the ML function representation is ensured by setting

$$
P_{S}^{\frac{\beta}{\alpha}} \ll P_{S}^{\frac{1-\alpha}{\alpha}}
$$

yielding

$$
P_{S}^{\frac{\beta-1+\alpha}{\alpha}} \ll 1
$$

We define the accuracy of the ML function representation by means of the parameter $\eta$,

$$
\eta=P_{S}^{\frac{\beta-1+\alpha}{\alpha}}
$$

For $0<\alpha<0.5$, Eq. (35) yields

$$
P_{S}=\eta
$$

while for $0.5 \leqslant \alpha<1$,

$$
P_{S}=\eta^{\frac{\alpha}{1-\alpha}}
$$


Equation (37) shows that the numerical accuracy of the ML function representation becomes more and more difficult to realize as $\alpha$ becomes closer and closer to the singularity $\alpha=1$.

Setting the accuracy $\eta$ of the ML function representation is equivalent to establishing the limited sensitivity of the event detector $P_{S}$, and, consequently, using Eq. (31) we can estimate the sensitivity of the ML complex process under study to noise. The process of detecting only a fraction of the PM map events, leading to the ML function with $\Lambda$ given by Eq. (24), once we scale it back to the original time scale, can be interpreted as the generator of a real ML function. This real ML function is close but not exactly identical to the ideal ML function (see Fig. 6), the accuracy of the ML function representation being determined by the nonvanishing parameter $\eta$. This prevents us from sending $P_{S}$ of Eq. (29) to zero, which would make the real ML process infinitely sensitive to noise. Of course, the same effect, of turning the survival probability $\Psi_{\epsilon}(t)$ into an extremely fast exponential decay, can be produced by increasing the value of $\epsilon$, namely, by increasing the noise intensity according to Eq. (17). This would have the effect of turning the ML function into an exponential function as shown from Fig. 7 for $\sigma=0.01$.

As far as the issue of the emergence of stretched exponential functions is concerned, we make the following prescription. In the absence of noise we determine $\alpha$ and $\lambda$ and their accuracy $\eta$ as a function of the detector sensitivity $P_{S}$. Then, on the basis of Eq. (29), we predict that the low value of $u$, at which the ML representation is invalidated, is given by

$$
u \approx \epsilon P_{S}^{-1 / \alpha} .
$$

If $\epsilon P_{S}^{-1 / \alpha} \approx \lambda$, the long-time tail of the ML function is erased and only the stretched exponential function is visible. When the condition $\epsilon P_{S}^{-1 / \alpha} \approx 1$ applies, noise has the effect of turning the complex survival probability into an exponential function. Thanks to Eq. (17) and to $\epsilon \approx 1 / t_{T}$, we can establish the value of noise intensity $\sigma$ making the survival probability $\Psi_{\epsilon}(t)$ virtually equivalent to a stretched exponential function.

\section{CONCLUDING REMARKS}

In the literature of complexity, some authors (see, for instance, Ref. [25]) refer to the emergence of stretched exponential functions as a form of complexity complementing the inverse power law distributions. This is a consequence of the fact that the stretched exponentials are frequently found in nature and economics [25].

In the subject of dielectric relaxation in solids $[26,27]$ the important issue has been debated for years of establishing a connection between the power law nature of dielectric response and dipole relaxation function. This issue was successfully addressed by Weron and Jurlewicz [28], who related the power law response function to the dipole relaxation function with the stretched exponential form.

As pointed out by Metzler and Klafter [14], for a long time there have been authors advocating the stretched exponential as well as authors advocating the inverse power law, as a statistical manifestation of complexity. The ML function, in a sense, can be interpreted as a bridge between these two apparently conflicting theoretical perspectives.
It is important to stress that this paper addresses this issue, as pointed out in Sec. I, from within the perspective of temporal complexity based on the occurrence of events. It confirms the conjectures made by the authors of earlier work [18,29], resting on the hypothesis that deviations from ordinary statistical physics are indeed due to the occurrence of crucial events. The authors of Ref. [18] interpreted their results as being the manifestation of a process driven by events and found stretched exponential functions, interpreted as the short-time manifestation of a ML function. This article confirms also the conjecture made by the authors of Ref. [29] that the stretched exponential revealed by their search of events in brain dynamics is the emerging tip of a ML function, the tails of which are killed by noise.

This paper shows that as an effect of noise the inverse power law of a ML function can become invisible and that only the short-time stretched exponential part of a ML function may remain visible when the noise intensity is very high. This might generate the impression that the stretched exponential function is an alternative sign of complexity. In conclusion, an important result of this article is its contribution to proving the universality of the ML function theoretical perspective.

There are numerical results on neural dynamics showing that the ML process may be generated by cooperation of limited intensity $[19,30]$. The results of this paper may be used to establish for which value of the cooperation parameter the distribution of the time distances between two consecutive neuron firings makes a transition from the exponential to the stretched exponential form.

\section{ACKNOWLEDGMENTS}

P.P., A.S., and P.G. warmly thank ARO and Welch for their support through Grants No. W911NF-11-1-0478 and No. B-1577, respectively.

\section{APPENDIX: NOTES ON THE EVALUATION OF THE HIGHER-ORDER TERMS}

We find $\hat{\psi}(u)$, the Laplace transform of

$$
\psi(t)=(\mu-1) \frac{T^{\mu-1}}{(t+T)^{\mu}}
$$

by means of the prescription

$$
\hat{\psi}(u)=\frac{(\mu-1) \Gamma(1-\mu)}{(u T)^{(1-\mu)}}\left[e^{u T}-E_{\mu-1}^{u T}\right],
$$

where

$$
E_{\mu-1}^{u T} \equiv \sum_{n=0}^{\infty} \frac{(u T)^{n+1-\mu}}{\Gamma(n+2-\mu)}
$$

is the generalized exponential function defined in Ref. [31]. By expanding the ordinary exponential $e^{u T}$ and using the expansion of Eq. (A3) we obtain

$$
\begin{aligned}
\hat{\psi}(u)= & {\left[1+\frac{\Gamma(2-\mu)}{\Gamma(3-\mu)} u T+\frac{\Gamma(2-\mu)}{\Gamma(4-\mu)}(u T)^{2}+\cdots\right] } \\
& -\Gamma(2-\mu)\left[(u T)^{\mu-1}+(u T)^{\mu}+\frac{1}{2}(u T)^{\mu+1}\right. \\
& \left.+\frac{1}{6}(u T)^{\mu+2}+\cdots\right] .
\end{aligned}
$$


Arranging terms according to increasing powers of $u$, we write

$$
\hat{\psi}(u)=1-\Gamma(2-\mu)(u T)^{\mu-1}+\Xi(u),
$$

where

$$
\Xi(u)=\frac{\Gamma(2-\mu)}{\Gamma(3-\mu)}(u T)-\Gamma(2-\mu)(u T)^{\mu}+\cdots .
$$

To estimate the error associated with the use of a finite rather than vanishing $P_{S}$ we consider the slowest contribution of order higher than $\mu-1$ in Eq. (A5). This leads us to estimate the order of the correction $\Sigma(u)$ of Eq. (21) by means of

$$
\Sigma(u)=\frac{T^{1-\alpha} u^{2-2 \alpha}}{\Gamma(2-\alpha)}-\Gamma(1-\alpha) T^{\alpha} u+\cdots .
$$

[1] Y. F. Contoyiannis, F. K. Diakonos, and A. Malakis, Phys. Rev. Lett. 89, 035701 (2002).

[2] Y. Pomeau and P. Manneville, Commun. Math. Phys. 74, 189 (1980).

[3] P. Gaspard and X.-J. Wang, Proc. Natl. Acad. Sci. USA 85, 4591 (1988).

[4] X.-J. Wang, Phys. Rev. A 40, 6647 (1989).

[5] S. Tasaki and P. Gaspard, J. Stat. Phys. 109, 803 (2002).

[6] T. Miyaguchi and Y. Aizawa, Phys. Rev. E 75, 066201 (2007).

[7] R. N. Mantegna and H. E. Stanley, Phys. Rev. Lett. 73, 2946 (1994).

[8] E. Barkai, Chem. Phys. 284, 13 (2002).

[9] A. von Kameke, F. Huhn, and V. Pérez-Munuzuri, Phys. Rev. E 85, 017201 (2012).

[10] E. G. Altmann and A. Endler, Phys. Rev. Lett. 105, 244102 (2010).

[11] E. G. Altmann and H. Kantz, Europhys. Lett. 78, 10008 (2007).

[12] R. Bettin, R. Mannella, B. J. West, and P. Grigolini, Phys. Rev. E 51, 212 (1995).

[13] E. Floriani, R. Mannella, and P. Grigolini, Phys. Rev. E 52, 5910 (1995).

[14] R. Metzler and J. Klafter, J. Non-Cryst. Solids 305, 81 (2002).

[15] R. L. Magin, C. Ingo, L. Colon-Perez, W. Trippett, and T. H. Mareci, Microporous Mesoporous Mater. 178, 39 (2013).

[16] F. Mainardi, Fractional Calculus and Waves in Linear Viscoelasticity (Imperial College Press, London, 2010).
[17] E. Lovecchio, P. Allegrini, E. Geneston, B. J. West, and P. Grigolini, Frontiers Physiol. 3, 98 (2012).

[18] R. Failla, P. Grigolini, M. Ignaccolo, and A. Schwettmann, Phys. Rev. E 70, 010101(R) (2004).

[19] M. Zare and P. Grigolini, Chaos Solitons Fractals 55, 80 (2013).

[20] P. Pramukkul, A. Svenkeson, P. Grigolini, M. Bologna, and B. J. West, Adv. Math. Phys. 2013, 498789 (2013).

[21] M. Ignaccolo, P. Grigolini, and A. Rosa, Phys. Rev. E 64, 026210 (2001).

[22] N. Korabel and E. Barkai, Phys. Rev. Lett. 102, 050601 (2009).

[23] S. Burov, R. Metzler, and E. Barkai, Proc. Natl. Acad. Sci. USA 107, 13228 (2010).

[24] D. Fulger, E. Scalas, and G. Germano, Phys. Rev. E 77, 021122 (2008).

[25] J. Laherrère and D. Sornette, Eur. Phys. J. B 2, 525 (1998).

[26] A. K. Jonscher, J. Phys. D: Appl. Phys. 32, R57 (1999).

[27] S. Havriliak, Jr. and S. J. Havriliak, Polymer 37, 4107 (1996).

[28] K. Weron and A. Jurlewicz, J. Phys. A: Math. Gen. 26, 395 (1993).

[29] S. Bianco, M. Ignaccolo, M. S. Rider, M. J. Ross, P. Winsor, and P. Grigolini, Phys. Rev. E 75, 061911 (2007).

[30] M. Zare and P. Grigolini, Phys. Rev. E 86, 051918 (2012).

[31] B. J. West, M. Bologna, and P. Grigolini, Physics of Fractal Operators (Springer Verlag, Berlin, 2003). 